\title{
Some Antiperiodic Boundary Value Problem for Nonlinear Fractional Impulsive Differential Equations
}

\author{
Xianghu Liu and Yanfang Li \\ School of Mathematics and Computer Science, Zunyi Normal College, Zunyi 563002, China \\ Correspondence should be addressed to Xianghu Liu; liouxianghu04@126.com
}

Received 1 January 2014; Accepted 22 March 2014; Published 16 June 2014

Academic Editor: Chuanzhi Bai

Copyright (C) 2014 X. Liu and Y. Li. This is an open access article distributed under the Creative Commons Attribution License, which permits unrestricted use, distribution, and reproduction in any medium, provided the original work is properly cited.

\begin{abstract}
This paper is concerned with the sufficient conditions for the existence of solutions for a class of generalized antiperiodic boundary value problem for nonlinear fractional impulsive differential equations involving the Riemann-Liouville fractional derivative. Firstly, we introduce the fractional calculus and give the generalized R-L fractional integral formula of R-L fractional derivative involving impulsive. Secondly, the sufficient condition for the existence and uniqueness of solutions is presented. Finally, we give some examples to illustrate our main results.
\end{abstract}

\section{Introduction}

Fractional calculus is a generalization of ordinary differentiation and integration; it is also as old as ordinary differential calculus. For the last decades, fractional differential equations have been receiving intensive attention because they provide an excellent tool for the description of memory and hereditary properties of various materials and processes, such as physics, mechanics, chemistry, and engineering; for more details, one can see Kilbas et al. [1] and Podlubny [2] and the references therein.

There have been considerable developments in the theory of impulsive differential equations in the last few decades. Impulsive differential equations have become more important in some mathematical models of real phenomena, especially in control, biology, medicine, and information (see [3, 4]). So the study of fractional impulsive differential equations is a more meaningful work. Some significant developments in fractional impulsive differential equations with Caputo derivative have been presented [5-27]. Recently, Fečkan et al. defined the solutions for fractional impulsive differential equations with Caputo derivative (for more details, see [17]). They considered the Cauchy problems for the following impulsive fractional differential equations:

$$
{ }^{c} D_{t}^{q} u(t)=f(t, u(t)), \quad t \in J^{\prime},
$$

$$
\begin{gathered}
u\left(t_{k}^{+}\right)=u\left(t_{k}^{-}\right)+y_{k}, \quad k=1, \ldots, m, \\
u(0)=u_{0},
\end{gathered}
$$

where $y_{k}(k=1, \ldots, m), u_{0}$ are constants. ${ }^{c} D_{t}^{q}(0<q<$ 1) denotes Caputo's fractional derivative. Some sufficient conditions for existence of the solutions have been established by applying Schaefer's fixed point theorem, Banach fixed point theorem, and the theorem of nonlinear alternative of Leray-Schauder type.

But as far as we know, there are few papers that consider the fractional impulsive differential equations with RiemannLiouville derivative (only see $[15,24]$ ).

Motivated by $[15,17,24]$ and some related literature, we study the existence and uniqueness of solutions for the generalized antiperiodic boundary value problem for fractional differential equations with impulsive effects

$$
\begin{gathered}
{ }^{L} D_{0^{+}}^{\alpha} u(t) \\
=f(t, u(t)), \quad t \in J, \quad t \neq t_{k}, k=1, \ldots, m, \\
\left.\Delta I_{0^{+}}^{1-\alpha} u(t)\right|_{t=t_{k}}=y_{k}, \quad k=1, \ldots, m, \\
\left.I_{0^{+}}^{1-\alpha} u(t)\right|_{t=0}=-\left.I_{0^{+}}^{1-\alpha} u(t)\right|_{t=T},
\end{gathered}
$$


where ${ }^{L} D_{0^{+}}^{\alpha}$ is the Riemann-Liouville fractional derivative, $f \in C(J \times R, R), J=(0, T], 0=t_{0}<t_{1}<\cdots<t_{m}<t_{m+1}=$ $T, y_{k} \in R$ are given constants and

$$
\left.\Delta I_{0^{+}}^{1-\alpha} u(t)\right|_{t=t_{k}}=\left.I_{0^{+}}^{1-\alpha} u(t)\right|_{t=t_{k}^{+}}-\left.I_{0^{+}}^{1-\alpha} u(t)\right|_{t=t_{k}^{-}},
$$

and $\left.I_{0^{+}}^{1-\alpha} u(t)\right|_{t=t_{k}^{-}}$and $\left.I_{0^{+}}^{1-\alpha} u(t)\right|_{t=t_{k}^{+}}$denote the left and the right limit of $I_{0^{+}}^{1-\alpha} u(t)$ at $t=t_{k}$, respectively.

For clarity and brevity, we restrict that the impulsive functions are constants $y_{k}, k=1, \ldots, m$. Indeed, we can also define the impulsive functions as $J_{k}\left(u\left(t_{k}\right)\right)\left(J_{k} \in C(R, R)\right)$.

Remark 1. For $\alpha=1$, (2) reduces to the first order nonlinear impulsive differential equation with antiperiodic boundary value problem.

To the best of the authors' knowledge, no one has studied the existence of solutions for (2). The purpose of this paper is to study the existence and uniqueness of solution of the generalized antiperiodic boundary value problem for nonlinear fractional impulsive differential equation involving Riemann-Liouville fractional derivative by using some fixed point theorems.

\section{Preliminaries and Lemmas}

In this section, we introduce notations, definitions, and preliminaries that will be used in this paper. In order to define the solution of (2), we will consider the following spaces.

$P C(J, R)=\left\{x: J \rightarrow R: x(t) \in C\left(t_{k}, t_{k+1}\right], k=0, \ldots, m ;\right.$ there exist $x\left(t_{k}^{+}\right)$and $x\left(t_{k}^{-}\right)$with $\left.x\left(t_{k}^{-}\right)=x\left(t_{k}\right), k=1, \ldots, m\right\}$.

$P C^{n}(J, R)=\left\{x \in P C^{n-1}(J, R): x^{(n)}(t) \in C\left(t_{k}, t_{k+1}\right]\right.$, $k=0, \ldots, m$; there exist $x^{(n)}\left(t_{k}^{+}\right) x^{(n)}\left(t_{k}^{-}\right)$with $x^{(n)}\left(t_{k}^{-}\right)=$ $\left.x^{(n)}\left(t_{k}\right), k=1, \ldots, m\right\}$.

$P C_{\gamma}(J, R)=\left\{x:\left.\left(t-t_{k}\right)^{\gamma} x\right|_{\left[t_{k}, t_{k+1}\right]} \in C\left[t_{k}, t_{k+1}\right], k=0, \ldots\right.$, $m\}$, where $0 \leq \gamma<1$.

It is easy to check that the space $P C_{\gamma}(J, R)$ is a Banach space with norm

$$
\|x\|_{P C_{\gamma}}=\sup _{t \in\left(t_{k}, t_{k+1}\right]}\left(t-t_{k}\right)^{\gamma}|x(t)|, \quad k=0, \ldots, m .
$$

Let us recall the following known definitions. For more details see [1].

Definition 2. Let $\Omega=[a, b](-\infty<a<b<\infty)$ be a finite interval on the real axis $R$. The Riemann-Liouville fractional integral of order $\alpha>0$ is defined by

$$
I_{0^{+}}^{\alpha} y(t)=\frac{1}{\Gamma(\alpha)} \int_{0}^{t}(t-s)^{\alpha-1} y(s) d s, \quad t>0 .
$$

Definition 3. The Riemann-Liouville derivative of order $\alpha>$ $0, n=[\alpha]+1$ can be written as

$$
\begin{aligned}
{ }^{L} D_{0^{+}}^{\alpha} y(t) & =\left(\frac{d}{d t}\right)^{n}\left(I_{0^{+}}^{n-\alpha} y(t)\right) \\
& =\frac{1}{\Gamma(n-\alpha)} \frac{d^{n}}{d t^{n}} \int_{0}^{t}(t-s)^{n-\alpha-1} y(s) d s, \quad t>0
\end{aligned}
$$

Lemma 4 (see Lemma 2.5 in [1]). Let $\alpha>0, n=[\alpha]+1$ and let $f_{n-\alpha}(t)=I_{0^{+}}^{n-\alpha} f(t)$ be the fractional integral of order $n-\alpha$. If $f(t) \in L_{1}(a, b)$ and $f_{n-\alpha}(t) \in C^{n}[a, b]$, we have the following equality:

$$
I_{0^{+}}^{\alpha L} D_{0^{+}}^{\alpha} f(t)=f(t)-\sum_{i=1}^{n} \frac{f_{n-\alpha}^{(n-i)}(0)}{\Gamma(\alpha-i+1)} t^{\alpha-i} .
$$

Lemma 5. If $a_{i}, b_{j} \neq 0(i=1,2, \ldots, k, j=0,1, \ldots, k-1)$, then

$$
\sum_{i=1}^{k} \sum_{j=0}^{i-1} a_{i} b_{j}=\sum_{j=0}^{k-1} \sum_{i=j+1}^{k} a_{i} b_{j},
$$

where $k \in N_{+}$.

Proof. If $k=1$, then we obtain

$$
a_{1} b_{0}=a_{1} b_{0} \text {. }
$$

Suppose $k=n$; the result holds; that is,

$$
\sum_{i=1}^{n} \sum_{j=0}^{i-1} a_{i} b_{j}=\sum_{j=0}^{n-1} \sum_{i=j+1}^{n} a_{i} b_{j} .
$$

When $k=n+1$, we obtain that

$$
\begin{aligned}
\sum_{i=1}^{n+1} \sum_{j=0}^{i-1} a_{i} b_{j} & =\sum_{i=1}^{n} \sum_{j=0}^{i-1} a_{i} b_{j}+a_{n+1} \sum_{j=0}^{n} b_{j} \\
& =\sum_{j=0}^{n-1} \sum_{i=j+1}^{n} a_{i} b_{j}+a_{n+1} \sum_{j=0}^{n} b_{j} \\
& =\sum_{j=0}^{n-1} \sum_{i=j+1}^{n+1} a_{i} b_{j}-a_{n+1} \sum_{j=0}^{n-1} b_{j}+a_{n+1} \sum_{j=0}^{n} b_{j} \\
& =\sum_{j=0}^{n-1} \sum_{i=j+1}^{n+1} a_{i} b_{j}+a_{n+1} b_{n}=\sum_{j=0}^{n} \sum_{i=j+1}^{n+1} a_{i} b_{j} .
\end{aligned}
$$

The proof is completed.

Lemma 6. Let $\alpha>0, n-1<\alpha<n, f_{n-\alpha}(t)=I_{0^{+}}^{n-\alpha} f(t)$. If $f(t) \in L_{1}(0, T)$ and $f_{n-\alpha}(t) \in P C^{n}(J, R)$, then for $t \in\left[0, t_{1}\right]$, one has

$$
I_{0^{+}}^{\alpha L} D_{0^{+}}^{\alpha} f(t)=f(t)-\sum_{i=1}^{n} \frac{f_{n-\alpha}^{(n-i)}(0)}{\Gamma(\alpha-i+1)} t^{\alpha-i} ;
$$

for $t \in\left(t_{k}, t_{k+1}\right], k=1, \ldots, m$, one has

$$
\begin{aligned}
I_{0^{+}}^{\alpha L} D_{0^{+}}^{\alpha} f(t)= & f(t)-\sum_{i=1}^{n} \frac{f_{n-\alpha}^{(n-i)}(0)}{\Gamma(\alpha-i+1)} t^{\alpha-i} \\
& -\sum_{j=1}^{k} \sum_{i=1}^{n} \frac{\Delta f_{n-\alpha}^{(n-i)}\left(t_{j}\right)}{\Gamma(\alpha-i+1)}\left(t-t_{j}\right)^{\alpha-i},
\end{aligned}
$$

where

$$
\Delta f_{n-\alpha}^{(n-i)}\left(t_{j}\right)=f_{n-\alpha}^{(n-i)}\left(t_{j}^{+}\right)-f_{n-\alpha}^{(n-i)}\left(t_{j}^{-}\right)
$$


Proof. Firstly, according to the fractional integral definitions, we get

$$
\begin{aligned}
I_{0^{+}}^{\alpha L} D_{0^{+}}^{\alpha} f(t) & \\
\quad= & \frac{1}{\Gamma(\alpha)} \int_{0}^{t}(t-s)^{\alpha-1}{ }^{L} D_{0^{+}}^{\alpha} f(s) d s \\
& =\frac{d}{d t}\left\{\frac{1}{\Gamma(\alpha+1)} \int_{0}^{t}(t-s)^{\alpha L} D_{0^{+}}^{\alpha} f(s) d s\right\} .
\end{aligned}
$$

If $t \in\left[0, t_{1}\right]$, by Lemma 4 , the result is easily to get.

If $t \in\left(t_{1}, t_{2}\right]$, integrating by parts repeatedly, we obtain

$$
\begin{aligned}
& \frac{1}{\Gamma(\alpha+1)} \int_{0}^{t}(t-s)^{\alpha L} D_{0^{+}}^{\alpha} f(s) d s \\
& =\frac{1}{\Gamma(\alpha+1)} \int_{0}^{t}(t-s)^{\alpha} \frac{d^{n}}{d s^{n}}\left\{I_{0^{+}}^{n-\alpha} f(s)\right\} d s \\
& =\frac{1}{\Gamma(\alpha+1) \Gamma(n-\alpha)} \\
& \times \int_{0}^{t}(t-s)^{\alpha} \frac{d^{n}}{d s^{n}} \int_{0}^{s}(s-\tau)^{n-\alpha-1} f(\tau) d \tau d s \\
& =\frac{1}{\Gamma(\alpha+1) \Gamma(n-\alpha)} \\
& \times \int_{0}^{t_{1}}(t-s)^{\alpha} \frac{d^{n}}{d s^{n}} \int_{0}^{s}(s-\tau)^{n-\alpha-1} f(\tau) d \tau d s \\
& +\frac{1}{\Gamma(\alpha+1) \Gamma(n-\alpha)} \\
& \times \int_{t_{1}}^{t}(t-s)^{\alpha} \frac{d^{n}}{d s^{n}} \int_{0}^{t_{1}}(s-\tau)^{n-\alpha-1} f(\tau) d \tau d s \\
& +\frac{1}{\Gamma(\alpha+1) \Gamma(n-\alpha)} \\
& \times \int_{t_{1}}^{t}(t-s)^{\alpha} \frac{d^{n}}{d s^{n}} \int_{t_{1}}^{s}(s-\tau)^{n-\alpha-1} f(\tau) d \tau d s \\
& =\frac{1}{\Gamma(\alpha-n+1) \Gamma(n-\alpha)} \\
& \times \int_{0}^{t_{1}}(t-s)^{\alpha-n} \int_{0}^{s}(s-\tau)^{n-\alpha-1} f(\tau) d \tau d s \\
& +\frac{1}{\Gamma(\alpha-i+2) \Gamma(n-\alpha)} \\
& \times\left.\sum_{i=1}^{n}\left[(t-s)^{\alpha-i+1} \frac{d^{n-i}}{d s^{n-i}} \int_{0}^{s}(s-\tau)^{n-\alpha-1} f(\tau) d \tau\right]\right|_{s=0} ^{s=t_{1}} \\
& +\frac{1}{\Gamma(\alpha-n+1) \Gamma(n-\alpha)} \\
& \times \int_{t_{1}}^{t}(t-s)^{\alpha-n} \int_{0}^{t_{1}}(s-\tau)^{n-\alpha-1} f(\tau) d \tau d s \\
& +\frac{1}{\Gamma(\alpha-i+2) \Gamma(n-\alpha)}
\end{aligned}
$$

$$
\begin{aligned}
& \times\left.\sum_{i=1}^{n}\left[(t-s)^{\alpha-i+1} \frac{d^{n-i}}{d s^{n-i}} \int_{0}^{t_{1}}(s-\tau)^{n-\alpha-1} f(\tau) d \tau\right]\right|_{s=t_{1}} ^{s=t} \\
& +\frac{1}{\Gamma(\alpha-n+1) \Gamma(n-\alpha)} \\
& \times \int_{t_{1}}^{t}(t-s)^{\alpha-n} \int_{t_{1}}^{s}(s-\tau)^{n-\alpha-1} f(\tau) d \tau d s \\
& +\frac{1}{\Gamma(\alpha-i+2) \Gamma(n-\alpha)} \\
& \times \sum_{i=1}^{n}\left[(t-s)^{\alpha-i+1} \frac{d^{n-i}}{d s^{n-i}}\right. \\
& \left.\times \int_{t_{1}}^{s}(s-\tau)^{n-\alpha-1} f(\tau) d \tau\right]\left.\right|_{s=t_{1}} ^{s=t} \\
& =\frac{1}{\Gamma(\alpha-n+1) \Gamma(n-\alpha)} \\
& \times \int_{0}^{t_{1}} f(\tau) d \tau \int_{\tau}^{t_{1}}(t-s)^{\alpha-n}(s-\tau)^{n-\alpha-1} d s \\
& +\frac{1}{\Gamma(\alpha-n+1) \Gamma(n-\alpha)} \\
& \times \int_{0}^{t_{1}} f(\tau) d \tau \int_{t_{1}}^{t}(t-s)^{\alpha-n}(s-\tau)^{n-\alpha-1} d s \\
& +\frac{1}{\Gamma(\alpha-n+1) \Gamma(n-\alpha)} \\
& \times \int_{t_{1}}^{t} f(\tau) d \tau \int_{\tau}^{t}(t-s)^{\alpha-n}(s-\tau)^{n-\alpha-1} d s \\
& -\sum_{i=1}^{n} \frac{f_{n-\alpha}^{(n-i)}(0)}{\Gamma(\alpha-i+2)} t^{\alpha-i+1} \\
& -\sum_{i=1}^{n} \frac{\Delta f_{n-\alpha}^{(n-i)}\left(t_{1}\right)}{\Gamma(\alpha-i+2)}\left(t-t_{1}\right)^{\alpha-i+1} \\
& =\int_{0}^{t} f(\tau) d \tau-\sum_{i=1}^{n} \frac{f_{n-\alpha}^{(n-i)}(0)}{\Gamma(\alpha-i+2)} t^{\alpha-i+1} \\
& -\sum_{i=1}^{n} \frac{\Delta f_{n-\alpha}^{(n-i)}\left(t_{1}\right)}{\Gamma(\alpha-i+2)}\left(t-t_{1}\right)^{\alpha-i+1},
\end{aligned}
$$

where the integral

$$
\begin{aligned}
& \int_{\tau}^{t}(t-s)^{\alpha-n}(s-\tau)^{n-\alpha-1} d s \\
& \quad=\int_{0}^{1}(1-z)^{\alpha-n} z^{n-\alpha-1} d z=B(\alpha-n+1, n-\alpha) \\
& \quad=\Gamma(\alpha-n+1) \Gamma(n-\alpha),
\end{aligned}
$$

where using the substitution $s=\tau+z(t-\tau)$. 
So, if $t \in\left(t_{1}, t_{2}\right]$, by (15), we have

$$
\begin{aligned}
I_{0^{+}}^{\alpha}{ }^{L} D_{0^{+}}^{\alpha} f(t)= & f(t)-\sum_{i=1}^{n} \frac{f_{n-\alpha}^{(n-i)}(0)}{\Gamma(\alpha-i+1)} t^{\alpha-i} \\
& -\sum_{i=1}^{n} \frac{\Delta f_{n-\alpha}^{(n-i)}\left(t_{1}\right)}{\Gamma(\alpha-i+1)}\left(t-t_{1}\right)^{\alpha-i} .
\end{aligned}
$$

If $t \in\left(t_{k}, t_{k+1}\right], k=2, \ldots, m$, integrating by parts and using Lemma 5 and (17) repeatedly, we get

$$
\begin{aligned}
& \frac{1}{\Gamma(\alpha+1)} \int_{0}^{t}(t-s)^{\alpha L} D_{0^{+}}^{\alpha} f(s) d s \\
& =\frac{1}{\Gamma(\alpha+1)} \int_{0}^{t}(t-s)^{\alpha} \frac{d^{n}}{d s^{n}}\left\{I_{0^{+}}^{n-\alpha} f(s)\right\} d s \\
& =\frac{1}{\Gamma(\alpha+1) \Gamma(n-\alpha)} \\
& \times \int_{0}^{t}(t-s)^{\alpha} \frac{d^{n}}{d s^{n}} \int_{0}^{s}(s-\tau)^{n-\alpha-1} f(\tau) d \tau d s \\
& =\frac{1}{\Gamma(\alpha+1) \Gamma(n-\alpha)} \\
& \times \int_{0}^{t_{1}}(t-s)^{\alpha} \frac{d^{n}}{d s^{n}} \int_{0}^{s}(s-\tau)^{n-\alpha-1} f(\tau) d \tau d s \\
& +\frac{1}{\Gamma(\alpha+1) \Gamma(n-\alpha)} \\
& \times \sum_{i=1}^{k-1} \int_{t_{i}}^{t_{i+1}}(t-s)^{\alpha} \frac{d^{n}}{d s^{n}} \\
& \times \sum_{j=0}^{i-1} \int_{t_{j}}^{t_{j+1}}(s-\tau)^{n-\alpha-1} f(\tau) d \tau d s \\
& +\frac{1}{\Gamma(\alpha+1) \Gamma(n-\alpha)} \\
& \times \sum_{i=1}^{k-1} \int_{t_{i}}^{t_{i+1}}(t-s)^{\alpha} \frac{d^{n}}{d s^{n}} \int_{t_{i}}^{s}(s-\tau)^{n-\alpha-1} f(\tau) d \tau d s \\
& +\frac{1}{\Gamma(\alpha+1) \Gamma(n-\alpha)} \\
& \times \int_{t_{k}}^{t}(t-s)^{\alpha} \frac{d^{n}}{d s^{n}} \sum_{j=0}^{k-1} \int_{t_{j}}^{t_{j+1}}(s-\tau)^{n-\alpha-1} f(\tau) d \tau d s \\
& +\frac{1}{\Gamma(\alpha+1) \Gamma(n-\alpha)} \\
& \times \int_{t_{k}}^{t}(t-s)^{\alpha} \frac{d^{n}}{d s^{n}} \int_{t_{k}}^{s}(s-\tau)^{n-\alpha-1} f(\tau) d \tau d s
\end{aligned}
$$

$$
\begin{aligned}
= & \frac{1}{\Gamma(\alpha-n+1) \Gamma(n-\alpha)} \\
& \times \int_{0}^{t_{1}}(t-s)^{\alpha-n} \int_{0}^{s}(s-\tau)^{n-\alpha-1} f(\tau) d \tau d s
\end{aligned}
$$$$
+\frac{1}{\Gamma(n-\alpha)}
$$$$
\times \sum_{p=1}^{n}\left[(t-s)^{\alpha-p+1} \frac{d^{n-p}}{d s^{n-p}}\right.
$$$$
\left.\times \int_{0}^{s}(s-\tau)^{n-\alpha-1} f(\tau) d \tau\right]\left.\right|_{s=0} ^{s=t_{1}}
$$$$
\times \frac{1}{\Gamma(\alpha-p+2)}
$$$$
+\frac{1}{\Gamma(\alpha+1) \Gamma(n-\alpha)}
$$$$
\times \sum_{i=1}^{k-1} \int_{t_{i}}^{t_{i+1}}(t-s)^{\alpha} \sum_{j=0}^{i-1} \int_{t_{j}}^{t_{j+1}}(s-\tau)^{n-\alpha-1} f(\tau) d \tau d s
$$$$
+\frac{1}{\Gamma(n-\alpha)}
$$$$
\times \sum_{i=1}^{k-1}\left\{\sum _ { p = 1 } ^ { n } \left[(t-s)^{\alpha-p+1} \frac{d^{n-p}}{d s^{n-p}}\right.\right.
$$$$
\times\left(\sum_{j=0}^{i-1} \int_{t_{j}}^{t_{j+1}}(s-\tau)^{n-\alpha-1}\right.
$$$$
\left.\times f(\tau) d \tau)]\left.\right|_{s=t_{i}} ^{s=t_{i+1}}\right\}
$$

$$
\times \frac{1}{\Gamma(\alpha-p+2)}
$$$$
+\frac{1}{\Gamma(\alpha+1) \Gamma(n-\alpha)}
$$$$
\times \sum_{i=1}^{k-1} \int_{t_{i}}^{t_{i+1}}(t-s)^{\alpha} \int_{t_{i}}^{s}(s-\tau)^{n-\alpha-1} f(\tau) d \tau d s
$$$$
+\frac{1}{\Gamma(n-\alpha)}
$$$$
\times \sum_{i=1}^{k-1}\left\{\sum _ { p = 1 } ^ { n } \left[(t-s)^{\alpha-p+1} \frac{d^{n-p}}{d s^{n-p}}\right.\right.
$$$$
\left.\left.\times \int_{t_{i}}^{s}(s-\tau)^{n-\alpha-1} f(\tau) d \tau\right]\left.\right|_{s=t_{i}} ^{s=t_{i+1}}\right\}
$$$$
\times \frac{1}{\Gamma(\alpha-p+2)}
$$ 


$$
\begin{aligned}
& +\frac{1}{\Gamma(\alpha+1) \Gamma(n-\alpha)} \\
& \times \int_{t_{k}}^{t}(t-s)^{\alpha} \sum_{j=0}^{k-1} \int_{t_{j}}^{t_{j+1}}(s-\tau)^{n-\alpha-1} f(\tau) d \tau d s \\
& +\frac{1}{\Gamma(n-\alpha)} \\
& \times \sum_{p=1}^{n}\left[(t-s)^{\alpha-p+1} \frac{d^{n-p}}{d s^{n-p}}\right. \\
& \left.\times\left(\sum_{j=0}^{k-1} \int_{t_{j}}^{t_{j+1}}(s-\tau)^{n-\alpha-1} f(\tau) d \tau\right)\right]\left.\right|_{s=t_{k}} ^{s=t} \\
& \times \frac{1}{\Gamma(\alpha-p+2)} \\
& +\frac{1}{\Gamma(\alpha+1) \Gamma(n-\alpha)} \\
& \times \int_{t_{k}}^{t}(t-s)^{\alpha} \int_{t_{k}}^{s}(s-\tau)^{n-\alpha-1} f(\tau) d \tau d s \\
& +\frac{1}{\Gamma(n-\alpha)} \\
& \times \sum_{p=1}^{n}\left[(t-s)^{\alpha-p+1} \frac{d^{n-p}}{d s^{n-p}}\right. \\
& \left.\times \int_{t_{k}}^{s}(s-\tau)^{n-\alpha-1} f(\tau) d \tau\right]\left.\right|_{s=t_{k}} ^{s=t} \\
& \times \frac{1}{\Gamma(\alpha-p+2)} \\
& =\frac{1}{\Gamma(\alpha-n+1) \Gamma(n-\alpha)} \\
& \times \int_{0}^{t_{1}} f(\tau) d \tau \int_{\tau}^{t_{1}}(t-s)^{\alpha-n}(s-\tau)^{n-\alpha-1} d s \\
& +\frac{1}{\Gamma(\alpha-n+1) \Gamma(n-\alpha)} \\
& \times \sum_{i=1}^{k-1} \sum_{j=0}^{i-1} \int_{t_{j}}^{t_{j+1}} f(\tau) d \tau \int_{t_{i}}^{t_{i+1}}(t-s)^{\alpha-n}(s-\tau)^{n-\alpha-1} d s \\
& +\frac{1}{\Gamma(\alpha-n+1) \Gamma(n-\alpha)} \\
& \times \sum_{i=1}^{k-1} \int_{t_{i}}^{t_{i+1}} f(\tau) d \tau \int_{\tau}^{t_{i+1}}(t-s)^{\alpha-n}(s-\tau)^{n-\alpha-1} d s \\
& +\frac{1}{\Gamma(\alpha-n+1) \Gamma(n-\alpha)} \\
& \times \sum_{j=0}^{k-1} \int_{t_{j}}^{t_{j+1}} f(\tau) d \tau \int_{t_{k}}^{t}(t-s)^{\alpha-n}(s-\tau)^{n-\alpha-1} d s
\end{aligned}
$$

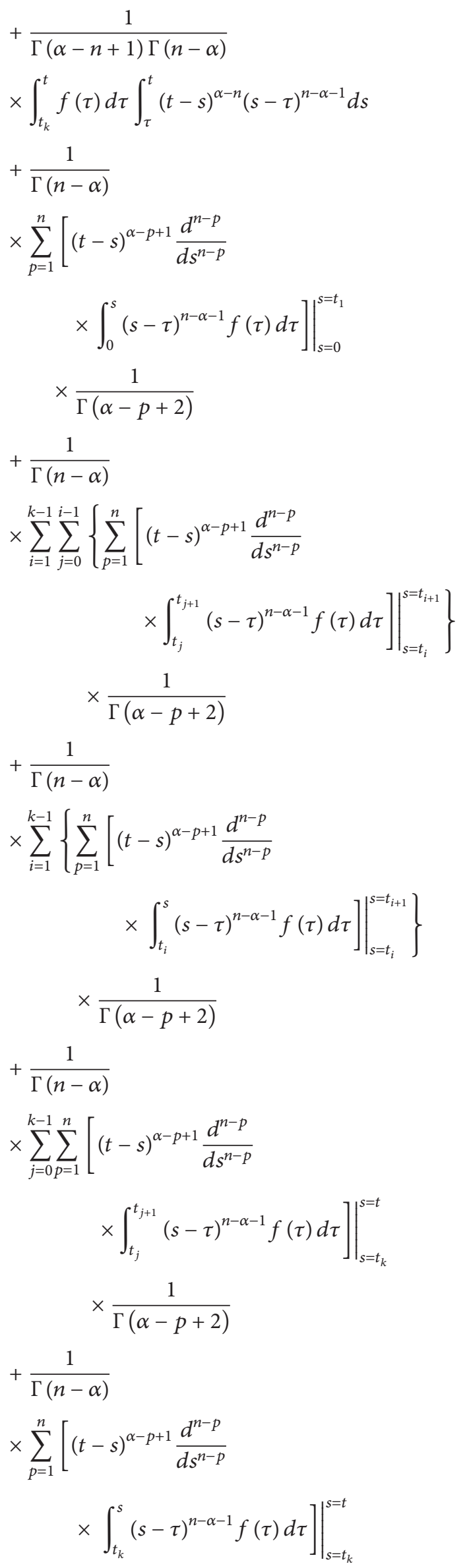




$$
\begin{aligned}
& \times \frac{1}{\Gamma(\alpha-p+2)} \\
& =\frac{1}{\Gamma(\alpha-n+1) \Gamma(n-\alpha)} \\
& \times \int_{0}^{t_{1}} f(\tau) d \tau \int_{\tau}^{t_{1}}(t-s)^{\alpha-n}(s-\tau)^{n-\alpha-1} d s \\
& +\frac{1}{\Gamma(\alpha-n+1) \Gamma(n-\alpha)} \\
& \times \sum_{j=0}^{k-2} \sum_{i=j+1}^{k-1} \int_{t_{j}}^{t_{j+1}} f(\tau) d \tau \int_{t_{i}}^{t_{i+1}}(t-s)^{\alpha-n}(s-\tau)^{n-\alpha-1} d s \\
& +\frac{1}{\Gamma(\alpha-n+1) \Gamma(n-\alpha)} \\
& \times \sum_{i=1}^{k-1} \int_{t_{i}}^{t_{i+1}} f(\tau) d \tau \int_{\tau}^{t_{i+1}}(t-s)^{\alpha-n}(s-\tau)^{n-\alpha-1} d s \\
& +\frac{1}{\Gamma(\alpha-n+1) \Gamma(n-\alpha)} \\
& \times \sum_{j=0}^{k-1} \int_{t_{j}}^{t_{j+1}} f(\tau) d \tau \int_{t_{k}}^{t}(t-s)^{\alpha-n}(s-\tau)^{n-\alpha-1} d s \\
& +\frac{1}{\Gamma(\alpha-n+1) \Gamma(n-\alpha)} \\
& \times \int_{t_{k}}^{t} f(\tau) d \tau \int_{\tau}^{t}(t-s)^{\alpha-n}(s-\tau)^{n-\alpha-1} d s \\
& +\frac{1}{\Gamma(n-\alpha)} \\
& \times \sum_{p=1}^{n}\left[(t-s)^{\alpha-p+1} \frac{d^{n-p}}{d s^{n-p}}\right. \\
& \left.\times \int_{0}^{s}(s-\tau)^{n-\alpha-1} f(\tau) d \tau\right]\left.\right|_{s=0} ^{s=t_{1}} \frac{1}{\Gamma(\alpha-p+2)} \\
& +\frac{1}{\Gamma(n-\alpha)} \\
& \times \sum_{i=1}^{k-1} \sum_{j=0}^{i-1}\left\{\sum _ { p = 1 } ^ { n } \left[(t-s)^{\alpha-p+1} \frac{d^{n-p}}{d s^{n-p}}\right.\right. \\
& \left.\left.\times \int_{t_{j}}^{t_{j+1}}(s-\tau)^{n-\alpha-1} f(\tau) d \tau\right]\left.\right|_{s=t_{i}} ^{s=t_{i+1}}\right\} \\
& \times \frac{1}{\Gamma(\alpha-p+2)} \\
& +\frac{1}{\Gamma(n-\alpha)} \\
& \times \sum_{i=1}^{k-1}\left\{\sum _ { p = 1 } ^ { n } \left[(t-s)^{\alpha-p+1} \frac{d^{n-p}}{d s^{n-p}}\right.\right. \\
& \left.\left.\times \int_{t_{i}}^{s}(s-\tau)^{n-\alpha-1} f(\tau) d \tau\right]\left.\right|_{s=t_{i}} ^{s=t_{i+1}}\right\}
\end{aligned}
$$

$$
\begin{aligned}
& \times \frac{1}{\Gamma(\alpha-p+2)} \\
& +\frac{1}{\Gamma(n-\alpha)} \\
& \times \sum_{j=0}^{k-1} \sum_{p=1}^{n}\left[(t-s)^{\alpha-p+1} \frac{d^{n-p}}{d s^{n-p}}\right. \\
& \left.\times \int_{t_{j}}^{t_{j+1}}(s-\tau)^{n-\alpha-1} f(\tau) d \tau\right]\left.\right|_{s=t_{k}} ^{s=t} \\
& \times \frac{1}{\Gamma(\alpha-p+2)} \\
& +\frac{1}{\Gamma(n-\alpha)} \\
& \times \sum_{p=1}^{n}\left[(t-s)^{\alpha-p+1} \frac{d^{n-p}}{d s^{n-p}}\right. \\
& \left.\times \int_{t_{k}}^{s}(s-\tau)^{n-\alpha-1} f(\tau) d \tau\right]\left.\right|_{s=t_{k}} ^{s=t} \\
& \times \frac{1}{\Gamma(\alpha-p+2)} \\
& =\int_{0}^{t} f(\tau) d \tau-\sum_{p=1}^{n} \frac{f_{n-\alpha}^{(n-p)}(0)}{\Gamma(\alpha-p+2)} t^{\alpha-p+1} \\
& -\sum_{i=1}^{k} \sum_{p=1}^{n} \frac{\Delta f_{n-\alpha}^{(n-p)}\left(t_{i}\right)}{\Gamma(\alpha-p+2)}\left(t-t_{i}\right)^{\alpha-p+1} .
\end{aligned}
$$

By (15), if $t \in\left(t_{k}, t_{k+1}\right], k=2, \ldots, m$, we have

$$
\begin{aligned}
I_{0^{+}}^{\alpha}{ }^{L} D_{0^{+}}^{\alpha} f(t)= & f(t)-\sum_{i=1}^{n} \frac{f_{n-\alpha}^{(n-i)}(0)}{\Gamma(\alpha-i+1)} t^{\alpha-i} \\
& -\sum_{j=1}^{k} \sum_{i=1}^{n} \frac{\Delta f_{n-\alpha}^{(n-i)}\left(t_{j}\right)}{\Gamma(\alpha-i+1)}\left(t-t_{j}\right)^{\alpha-i}
\end{aligned}
$$

The proof is completed.

Remark 7. In Lemma 6, if the assumption $f_{n-\alpha}(t) \in P C^{n}(J, R)$ is replaced by $f_{n-\alpha}(t) \in C^{n}(J, R)$, we will get the same result of Lemma 4.

Lemma 8. The impulsive antiperiodic boundary value problem

$$
\begin{aligned}
& { }^{L} D_{0^{+}}^{\alpha} u(t)=\rho(t), \\
& 0<\alpha \leq 1, t \in J, \quad t \neq t_{k}, k=1, \ldots, m, \\
& \left.\Delta I_{0^{+}}^{1-\alpha} u(t)\right|_{t=t_{k}}=y_{k}, \quad k=1, \ldots, m, \\
& \left.\quad I_{0^{+}}^{1-\alpha} u(t)\right|_{t=0}=-\left.I_{0^{+}}^{1-\alpha} u(t)\right|_{t=T},
\end{aligned}
$$


where $\rho(t) \in C(J, R)$, has a unique solution $u(t) \in P C_{1-\alpha}(J, R)$ given by

$u(t)$

$$
\left\{\begin{array}{l}
\frac{a}{\Gamma(\alpha)} t^{\alpha-1}+\frac{1}{\Gamma(\alpha)} \\
\quad \times \int_{0}^{t}(t-s)^{\alpha-1} \rho(s) d s, \quad t \in\left(0, t_{1}\right] \\
\frac{a}{\Gamma(\alpha)} t^{\alpha-1} \\
+\sum_{0<t_{i}<t} \frac{y_{i}}{\Gamma(\alpha)}\left(t-t_{i}\right)^{\alpha-1} \\
+\frac{1}{\Gamma(\alpha)} \\
\quad \times \int_{0}^{t}(t-s)^{\alpha-1} \rho(s) d s, \quad t \in\left(t_{k}, t_{k+1}\right], k=1,2, \ldots, m,
\end{array}\right.
$$

where

$$
a=-\frac{1}{2}\left(\sum_{i=1}^{m} y_{i}+\int_{0}^{T} \rho(s) d s\right) .
$$

Proof. Let $u(t)$ be a solution of (21). By Lemma $6(n=1)$, we have

$u(t)$

$$
\left\{\begin{array}{l}
\frac{a}{\Gamma(\alpha)} t^{\alpha-1}+\frac{1}{\Gamma(\alpha)} \\
\quad \times \int_{0}^{t}(t-s)^{\alpha-1} \rho(s) d s, \quad t \in\left(0, t_{1}\right] \\
\frac{a}{\Gamma(\alpha)} t^{\alpha-1} \\
+\sum_{0<t_{i}<t} \frac{y_{i}}{\Gamma(\alpha)}\left(t-t_{i}\right)^{\alpha-1} \\
+\frac{1}{\Gamma(\alpha)} \\
\times \int_{0}^{t}(t-s)^{\alpha-1} \rho(s) d s, \quad t \in\left(t_{k}, t_{k+1}\right], k=1,2, \ldots, m,
\end{array}\right.
$$

where $\left.I_{0^{+}}^{1-\alpha} u(t)\right|_{t=0}=a$.

According to the following properties:

$$
\begin{gathered}
I_{0^{+}}^{1-\alpha} t^{\alpha-1}=\Gamma(\alpha), \\
I_{0^{+}}^{1-\alpha} \sum_{0<t_{i}<t} y_{i}\left(t-t_{i}\right)^{\alpha-1} \\
=\frac{1}{\Gamma(1-\alpha)} \int_{0}^{t}(t-s)^{-\alpha} \sum_{0<t_{i}<s} y_{i}\left(s-t_{i}\right)^{\alpha-1} d s \\
=\frac{1}{\Gamma(1-\alpha)} \int_{t_{i}}^{t}(t-s)^{-\alpha} \sum_{0<t_{i}<s} y_{i}\left(s-t_{i}\right)^{\alpha-1} d s \\
=\Gamma(\alpha) \sum_{0<t_{i}<t} y_{i},
\end{gathered}
$$

we obtain

$$
\begin{aligned}
& I_{0^{+}}^{1-\alpha} u(t) \\
& =\left\{\begin{array}{l}
a+\int_{0}^{t} \rho(s) d s, \quad t \in\left(0, t_{1}\right], \\
a+\sum_{0<t_{i}<t} y_{i} \\
\quad+\int_{0}^{t} \rho(s) d s, \quad t \in\left(t_{k}, t_{k+1}\right], k=1,2, \ldots, m .
\end{array}\right.
\end{aligned}
$$

Then by the antiperiodic boundary value condition, we have

$$
a=-\frac{1}{2}\left(\sum_{i=1}^{m} y_{i}+\int_{0}^{T} \rho(s) d s\right) .
$$

Conversely, assuming that $u(t)$ is a solution of the impulsive fractional integral equation (22), we can obtain the impulsive fractional differential equation (21).

This completes the proof.

\section{Main Results}

This section deals with the existence and uniqueness of solutions for the problem (2).

Firstly, for $t \in\left(t_{k}, t_{k+1}\right], k=0,1, \ldots, m$, we define an operator $T$ as

$$
\begin{aligned}
(T u)(t)= & -\frac{t^{\alpha-1}}{2 \Gamma(\alpha)}\left(\sum_{i=1}^{m} y_{i}+\int_{0}^{T} f(s, u(s)) d s\right) \\
& +\sum_{0<t_{i}<t} \frac{y_{i}}{\Gamma(\alpha)}\left(t-t_{i}\right)^{\alpha-1} \\
& +\frac{1}{\Gamma(\alpha)} \int_{0}^{t}(t-s)^{\alpha-1} f(s, u(s)) d s .
\end{aligned}
$$

Theorem 9. If the following condition is satisfied:

(H1): there exist constants $\ell, N>0$ such that

$$
\begin{array}{r}
|f(t, u)| \leq \ell+N\|u\|^{\theta}, \quad \forall t \in J, \\
u \in R, \quad 0 \leq \theta<1,
\end{array}
$$

then the fractional impulsive differential equation (2) has at least one solution.

Proof. Assume (H1) hold; let

$$
\begin{aligned}
& \lambda=\max \left\{\frac{9}{2 \Gamma(\alpha)} \sum_{i=1}^{m}\left|y_{i}\right|,\right. \\
& {\left[3\left(\frac{1}{2 \Gamma(\alpha)}+\frac{1}{\Gamma(\alpha+1)}\right) N T\right]^{1 /(1-\theta)}, } \\
&\left.3\left(\frac{1}{2 \Gamma(\alpha)}+\frac{1}{\Gamma(\alpha+1)}\right) \ell T\right\}
\end{aligned}
$$


and define $A_{1-\alpha}^{\lambda}=\left\{u \in P C_{1-\alpha}(J, R):\|u\| \leq \lambda\right\}$.

When $t \in\left(t_{k}, t_{k+1}\right], k=0,1, \ldots, m$, for $\forall u \in A_{1-\alpha}^{\lambda}$, by (H1), we have

$$
\begin{aligned}
(t- & \left.t_{k}\right)^{1-\alpha}|(T u)(t)| \\
\leq & \frac{\left(t-t_{k}\right)^{1-\alpha} t^{\alpha-1}}{2 \Gamma(\alpha)} \\
& \times \sum_{i=1}^{m}\left|y_{i}\right|+\frac{\left(t-t_{k}\right)^{1-\alpha} t^{\alpha-1}}{2 \Gamma(\alpha)} \int_{0}^{T}|f(s, u(s))| d s \\
& +\frac{\left(t-t_{k}\right)^{1-\alpha}}{\Gamma(\alpha)} \sum_{0<t_{i}<t}\left|y_{i}\right|\left(t-t_{i}\right)^{\alpha-1} \\
& +\frac{\left(t-t_{k}\right)^{1-\alpha}}{\Gamma(\alpha)} \int_{0}^{t}(t-s)^{\alpha-1}|f(s, u(s))| d s \\
\leq & \frac{3}{2 \Gamma(\alpha)} \sum_{i=1}^{m}\left|y_{i}\right|+\frac{T}{2 \Gamma(\alpha)}\left(\ell+N \lambda^{\theta}\right) \\
= & \frac{3}{2 \Gamma(\alpha)} \sum_{i=1}^{m}\left|y_{i}\right|+\left(\frac{1}{2 \Gamma(\alpha)}+\frac{1}{\Gamma(\alpha+1)}\right) N T \lambda^{\theta} \\
& +\left(\frac{1}{2 \Gamma(\alpha)}+\frac{1}{\Gamma(\alpha+1)}\right) \ell T \leq \lambda, \\
& \left.+\ell+N \lambda^{\theta}\right) \\
&
\end{aligned}
$$

which implies that $T: A_{1-\alpha}^{\lambda} \rightarrow A_{1-\alpha}^{\lambda}$.

In view of the continuity of $f$, we get that the operator $T$ is continuous easily.

Next, we will prove that $T$ is a completely continuous operator.

For $\left(t_{k}, t_{k+1}\right], k=0, \ldots, m$, if $t_{k}<\tau_{1}<\tau_{2} \leq t_{k+1}, u \in$ $A_{1-\alpha}^{\lambda}$, when $\tau_{1} \rightarrow \tau_{2}$, by (H1), we have

$$
\begin{aligned}
\mid\left(\tau_{1}-t_{k}\right)^{1-\alpha}(T u)\left(\tau_{1}\right) \\
-\left(\tau_{2}-t_{k}\right)^{1-\alpha}(T u)\left(\tau_{2}\right) \mid \\
\leq \frac{\left|\left(\tau_{1}-t_{k}\right)^{1-\alpha} \tau_{1}^{\alpha-1}-\left(\tau_{2}-t_{k}\right)^{1-\alpha} \tau_{2}^{\alpha-1}\right|}{2 \Gamma(\alpha)} \\
\quad \times \sum_{i=1}^{m}\left|y_{i}\right| \\
+\frac{\left|\left(\tau_{1}-t_{k}\right)^{1-\alpha} \tau_{1}^{\alpha-1}-\left(\tau_{2}-t_{k}\right)^{1-\alpha} \tau_{2}^{\alpha-1}\right|}{2 \Gamma(\alpha)} \\
\quad \times \int_{0}^{T}|f(s, u(s))| d s \\
+\mid \frac{\left(\tau_{1}-t_{k}\right)^{1-\alpha}}{\Gamma(\alpha)} \sum_{0<t_{i}<t} y_{i}\left(\tau_{1}-t_{i}\right)^{\alpha-1} \\
\quad-\frac{\left(\tau_{2}-t_{k}\right)^{1-\alpha}}{\Gamma(\alpha)} \sum_{0<t_{i}<t} y_{i}\left(\tau_{2}-t_{i}\right)^{\alpha-1} \mid
\end{aligned}
$$

$$
\begin{aligned}
& +\frac{\left|\left(\tau_{1}-t_{k}\right)^{1-\alpha}-\left(\tau_{2}-t_{k}\right)^{1-\alpha}\right|}{\Gamma(\alpha)} \\
& \times \int_{0}^{t}(t-s)^{\alpha-1}|f(s, u(s))| d s \\
& \leq \frac{\left|\left(1-t_{k} / \tau_{1}\right)^{1-\alpha}-\left(1-t_{k} / \tau_{2}\right)^{1-\alpha}\right|}{2 \Gamma(\alpha)} \sum_{i=1}^{m}\left|y_{i}\right| \\
& +\frac{\left|\left(1-t_{k} / \tau_{1}\right)^{1-\alpha}-\left(1-t_{k} / \tau_{2}\right)^{1-\alpha}\right|}{2 \Gamma(\alpha)} \\
& \times\left(\ell+N \lambda^{\theta}\right) T+\frac{1}{\Gamma(\alpha)} \\
& \times \mid \sum_{i=1}^{k-1} y_{i}\left(\tau_{1}-t_{i}\right)^{\alpha-1} \\
& +\frac{\left(\ell+N \lambda^{\theta}\right) T^{\alpha} \longrightarrow 0 .}{\Gamma(\alpha+1)} \\
& +\frac{\left|\left(\tau_{1}-t_{k}\right)^{1-\alpha}-\left(\tau_{2}-t_{k}\right)^{1-\alpha}\right|}{k-1} y_{i}\left(\tau_{2}-t_{i}\right)^{\alpha-1} \mid
\end{aligned}
$$

According to the Ascoli-Arzela theorem, we can obtain $T: A_{1-\alpha}^{\lambda} \rightarrow A_{1-\alpha}^{\lambda}$ which is a completely continuous operator. Therefore, by Schauder's fixed point theorem, the operator $T$ has at least one fixed point, which implies that fractional impulsive differential equation (2) has at least one solution $u(t)$.

Theorem 10. Assume that

(H2): there exists constant $L \geq 0$ such that

$$
\left|f\left(t, u_{1}\right)-f\left(t, u_{2}\right)\right| \leq L\left\|u_{1}-u_{2}\right\|, \quad \forall u_{1}, u_{2} \in P C_{\gamma}(J, R) .
$$

Then problem (2) has a unique solution if

$$
\left(\frac{1}{2 \Gamma(\alpha)}+\frac{1}{\Gamma(\alpha+1)}\right) L T<1 .
$$

Proof. We define $\sup _{t \in J}|f(t, 0)|=M$ and choose

$$
r=\frac{(3 / 2 \Gamma(\alpha)) \sum_{i=1}^{m}\left|y_{i}\right|+(1 / 2 \Gamma(\alpha)+1 / \Gamma(\alpha+1)) M T}{1-(1 / 2 \Gamma(\alpha)+1 / \Gamma(\alpha+1)) L T} .
$$

Firstly, we prove that $T u \in B_{1-\alpha}^{r}$, where $B_{1-\alpha}^{r}=\{u \in$ $\left.P C_{1-\alpha}(J, R):\|u\| \leq r\right\}$.

For $\forall u \in B_{1-\alpha}^{r}$, by (H2), we have

$$
\begin{aligned}
& \left(t-t_{k}\right)^{1-\alpha}|(T u)(t)| \\
& \leq \frac{\left(t-t_{k}\right)^{1-\alpha} t^{\alpha-1}}{2 \Gamma(\alpha)} \sum_{i=1}^{m}\left|y_{i}\right|
\end{aligned}
$$




$$
\begin{aligned}
& +\frac{\left(t-t_{k}\right)^{1-\alpha} t^{\alpha-1}}{2 \Gamma(\alpha)} \int_{0}^{T}|f(s, u(s))| d s \\
& +\frac{\left(t-t_{k}\right)^{1-\alpha}}{\Gamma(\alpha)} \\
& \times\left(\sum_{0<t_{i}<t}\left|y_{i}\right|\left(t-t_{i}\right)^{\alpha-1}\right. \\
& \left.+\int_{0}^{t}(t-s)^{\alpha-1}|f(s, u(s))| d s\right) \\
& \leq \frac{3}{2 \Gamma(\alpha)} \sum_{i=1}^{m}\left|y_{i}\right|+\frac{1}{2 \Gamma(\alpha)} \\
& \times \int_{0}^{T}(|f(s, u(s))-f(s, 0)|+|f(s, 0)|) d s \\
& +\frac{\left(t-t_{k}\right)^{1-\alpha}}{\Gamma(\alpha)} \\
& \times \int_{0}^{t}(t-s)^{\alpha-1} \\
& \times(|f(s, u(s))-f(s, 0)|+|f(s, 0)|) d s \\
& \leq \frac{3}{2 \Gamma(\alpha)} \sum_{i=1}^{m}\left|y_{i}\right|+\frac{\operatorname{LTr}}{2 \Gamma(\alpha)} \\
& +\frac{M T}{2 \Gamma(\alpha)}+\frac{L T r}{\Gamma(\alpha+1)}+\frac{M T}{\Gamma(\alpha+1)} \\
& =\frac{3}{2 \Gamma(\alpha)} \sum_{i=1}^{m}\left|y_{i}\right|+\left(\frac{1}{2 \Gamma(\alpha)}+\frac{1}{\Gamma(\alpha+1)}\right) L T r \\
& +\left(\frac{1}{2 \Gamma(\alpha)}+\frac{1}{\Gamma(\alpha+1)}\right) M T=r .
\end{aligned}
$$

Next, for $\forall u, v \in B_{1-\alpha}^{r}$, by (H2), we get

$$
\begin{aligned}
& \left(t-t_{k}\right)^{1-\alpha}|(T u)(t)-(T v)(t)| \\
& \leq \frac{\left(t-t_{k}\right)^{1-\alpha} t^{\alpha-1}}{2 \Gamma(\alpha)} \\
& \quad \times \int_{0}^{T}|f(s, u(s))-f(s, v(s))| d s \\
& \quad+\frac{\left(t-t_{k}\right)^{1-\alpha}}{\Gamma(\alpha)} \\
& \quad \times \int_{0}^{t}(t-s)^{\alpha-1}(|f(s, u(s))-f(s, v(s))|) d s \\
& \leq\left(\frac{L T}{2 \Gamma(\alpha)}+\frac{L T}{\Gamma(\alpha+1)}\right)\|u-v\|_{B_{1-\alpha}^{r} .}
\end{aligned}
$$

According to inequality (34), we obtain that the operator $T$ is a contractive mapping on $B_{1-\alpha}^{r}$. Hence, by Banach fixed point theorem, problem (2) has a unique solution.

The proof is completed.

\section{Examples}

Example 1. Choose $\alpha=1 / 2, t_{1}=1 / 2$, and $T=1$, and consider the following fractional impulsive generalized antiperiodic boundary value problem:

$$
\begin{gathered}
{ }^{L} D_{0^{+}}^{1 / 2} u(t)=f(t, u(t)), \quad t \neq \frac{1}{2}, t \in[0,1] \\
\Delta u\left(\frac{1}{2}\right)=b, \quad b \in R \\
\left.I_{0^{+}}^{1 / 2} u(t)\right|_{t=0}=-\left.I_{0^{+}}^{1 / 2} u(t)\right|_{t=1}
\end{gathered}
$$

where

$$
f(t, u(t))=\sin t+e^{-t} \sqrt{t u(t)} .
$$

Let $\ell=N=1, \theta=1 / 2$; clearly, assumption $(\mathrm{H} 1)$ is satisfied. By Theorem 9, the fractional impulsive generalized antiperiodic boundary value problem (38) has at least one solution.

Example 2. Choose $\alpha=2 / 3, t_{1}=1 / 2$, and $T=1$; consider the following fractional impulsive generalized antiperiodic boundary value problem:

$$
\begin{gathered}
{ }^{L} D_{0^{+}}^{2 / 3} u(t)=f(t, u(t)), \quad t \neq \frac{1}{2}, t \in[0,1], \\
\Delta u\left(\frac{1}{2}\right)=b, \quad b \in R \\
\left.I_{0^{+}}^{2 / 3} u(t)\right|_{t=0}=-\left.I_{0^{+}}^{2 / 3} u(t)\right|_{t=1},
\end{gathered}
$$

where

$$
f(t, u(t))=t^{3}+\frac{\Gamma(2 / 3) \sin t}{3 e^{t^{1 / 2}}} t^{1 / 3} u(t) .
$$

Letting $L=\Gamma(2 / 3) / 3$, condition $(\mathrm{H} 2)$ of Theorem 10 can be verified, so Example 2 has at least one solution.

\section{Conflict of Interests}

The authors declare that there is no conflict of interests regarding the publication of this paper.

\section{Acknowledgments}

The authors thank the referees for their careful reading of the paper and insightful comments that will improve the quality of the paper. They would also like to acknowledge the valuable comments and suggestions from the editors that will vastly contribute to the improvement of the presentation of the paper. This project is supported by NNSF of China Grant 
nos. 11271087 and 61263006, Guangxi Scientific Experimental (China-ASEAN Research) Centre no. 20120116, the Open Fund of Guangxi Key Laboratory of Hybrid Computation, IC Design Analysis no. 2012HCIC07, and the Guizhou Province Science and Technology Fund (nos. LKZS[2014]29 and LKZS[2014]30).

\section{References}

[1] A. A. Kilbas, H. M. Srivastava, and J. J. Trujillo, Theory and Applications of Fractional Differential Equations, Elsevier, Amsterdam, The Netherlands, 2006.

[2] I. Podlubny, Fractional Differential Equations, vol. 198 of Mathematics in Science and Engineering, Academic Press, New York, NY, USA, 1999.

[3] M. Benchohra, J. Henderson, and S. Ntouyas, Impulsive Differential Equations and Inclusions, Hindawi Publishing Corporation, New York, NY, USA, 2006.

[4] V. Lakshmikantham, D. D. Baĭnov, and P. S. Simeonov, Theory of Impulsive Differential Equations, World Scientific, Singapore, 1989.

[5] A. Anguraj and P. Karthikeyan, "Anti-periodic boundary value problem for impulsive fractional integro differential equations," Fractional Calculus \& Applied Analysis, vol. 13, no. 3, pp. 281293, 2010.

[6] B. Ahmad and S. Sivasundaram, "Existence of solutions for impulsive integral boundary value problems of fractional order," Nonlinear Analysis: Hybrid Systems, vol. 4, no. 1, pp. 134141, 2010.

[7] B. Ahmad and S. Sivasundaram, "Existence results for nonlinear impulsive hybrid boundary value problems involving fractional differential equations," Nonlinear Analysis: Hybrid Systems, vol. 3, no. 3, pp. 251-258, 2009.

[8] B. Ahmad and G. Wang, "A study of an impulsive four-point nonlocal boundary value problem of nonlinear fractional differential equations," Computers \& Mathematics with Applications, vol. 62, no. 3, pp. 1341-1349, 2011.

[9] K. Balachandran and S. Kiruthika, "Existence of solutions of abstract fractional impulsive semilinear evolution equations," Electronic Journal of Qualitative Theory of Differential Equations, no. 4, pp. 1-12, 2010.

[10] K. Balachandran, S. Kiruthika, and J. J. Trujillo, "Existence results for fractional impulsive integrodifferential equations in Banach spaces," Communications in Nonlinear Science and Numerical Simulation, vol. 16, no. 4, pp. 1970-1977, 2011.

[11] K. Balachandran, S. Kiruthika, and J. J. Trujillo, “On fractional impulsive equations of Sobolev type with nonlocal condition in Banach spaces," Computers \& Mathematics with Applications, vol. 62 , no. 3, pp. 1157-1165, 2011.

[12] M. Benchohra, S. Hamani, J. J. Nieto, and B. A. Slimani, "Existence of solutions to differential inclusions with fractional order and impulses," Electronic Journal of Differential Equations, no. 80, pp. 1-18, 2010.

[13] M. Benchohra and B. A. Slimani, "Existence and uniqueness of solutions to impulsive fractional differential equations," Electronic Journal of Differential Equations, vol. 2009, pp. 1-11, 2009.

[14] M. Benchohra and S. Hamani, "The method of upper and lower solutions and impulsive fractional differential inclusions," Nonlinear Analysis: Hybrid Systems, vol. 3, no. 4, pp. 433-440, 2009.
[15] C. Bai, "Impulsive periodic boundary value problems for fractional differential equation involving Riemann-Liouville sequential fractional derivative," Journal of Mathematical Analysis and Applications, vol. 384, no. 2, pp. 211-231, 2011.

[16] F. Chen, A. Chen, and X. Wang, "On the solutions for impulsive fractional functional differential equations," Differential Equations and Dynamical Systems, vol. 17, no. 4, pp. 379-391, 2009.

[17] M. Fečkan, Y. Zhou, and J. Wang, "On the concept and existence of solution for impulsive fractional differential equations," Communications in Nonlinear Science and Numerical Simulation, vol. 17, no. 7, pp. 3050-3060, 2012.

[18] L. Yang and H. Chen, "Nonlocal boundary value problem for impulsive differential equations of fractional order," Advances in Difference Equations, vol. 2011, Article ID 404917, 16 pages, 2011.

[19] X. B. Shu, Y. Lai, and Y. Chen, "The existence of mild solutions for impulsive fractional partial differential equations," Nonlinear Analysis: Theory, Methods \& Applications, vol. 74, no. 5, pp. 2003-2011, 2011.

[20] Y. S. Tian and Z. B. Bai, "Existence results for the three-point impulsive boundary value problem involving fractional differential equations," Computers \& Mathematics with Applications, vol. 59, no. 8, pp. 2601-2609, 2010.

[21] G. T. Wang, B. Ahmad, and L. H. Zhang, "Impulsive antiperiodic boundary value problem for nonlinear differential equations of fractional order," Nonlinear Analysis: Theory, Methods \& Applications, vol. 74, no. 3, pp. 792-804, 2011.

[22] G. T. Wang, B. Ahmad, and L. Zhang, "Some existence results for impulsive nonlinear fractional differential equations with mixed boundary conditions," Computers \& Mathematics with Applications, vol. 62, no. 3, pp. 1389-1397, 2011.

[23] H. Wang, "Existence results for fractional functional differential equations with impulses," Journal of Applied Mathematics and Computing, vol. 38, no. 1-2, pp. 85-101, 2012.

[24] X. J. Wang and C. Z. Bai, "Periodic boundary value problems for nonlinear impulsive fractional differential equation," Electronic Journal of Qualitative Theory of Differential Equations, no. 3, pp. $1-15,2011$.

[25] J. R. Wang, M. Fečkan, and Y. Zhou, "On the new concept of solutions and existence results for impulsive fractional evolution equations," Dynamics of Partial Differential Equations, vol. 8, no. 4, pp. 345-361, 2011.

[26] L. Zhang and G. Wang, "Existence of solutions for nonlinear fractional differential equations with impulses and antiperiodic boundary conditions," Electronic Journal of Qualitative Theory of Differential Equations, no. 7, pp. 1-11, 2011.

[27] G. Wang, B. Ahmad, L. Zhang, and J. J. Nieto, "Comments on the concept of existence of solution for impulsive fractional differential equations," Communications in Nonlinear Science and Numerical Simulation, vol. 19, no. 3, pp. 401-403, 2014. 


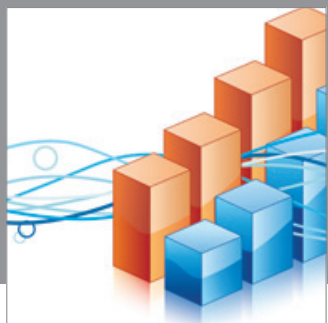

Advances in

Operations Research

mansans

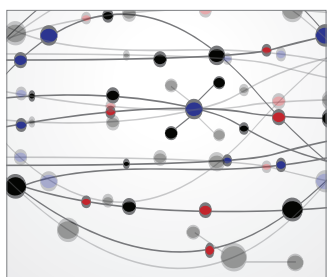

The Scientific World Journal
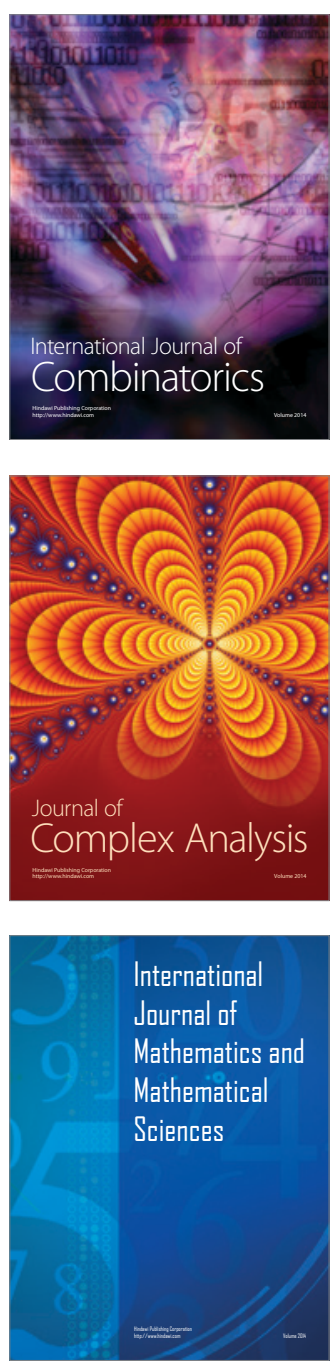
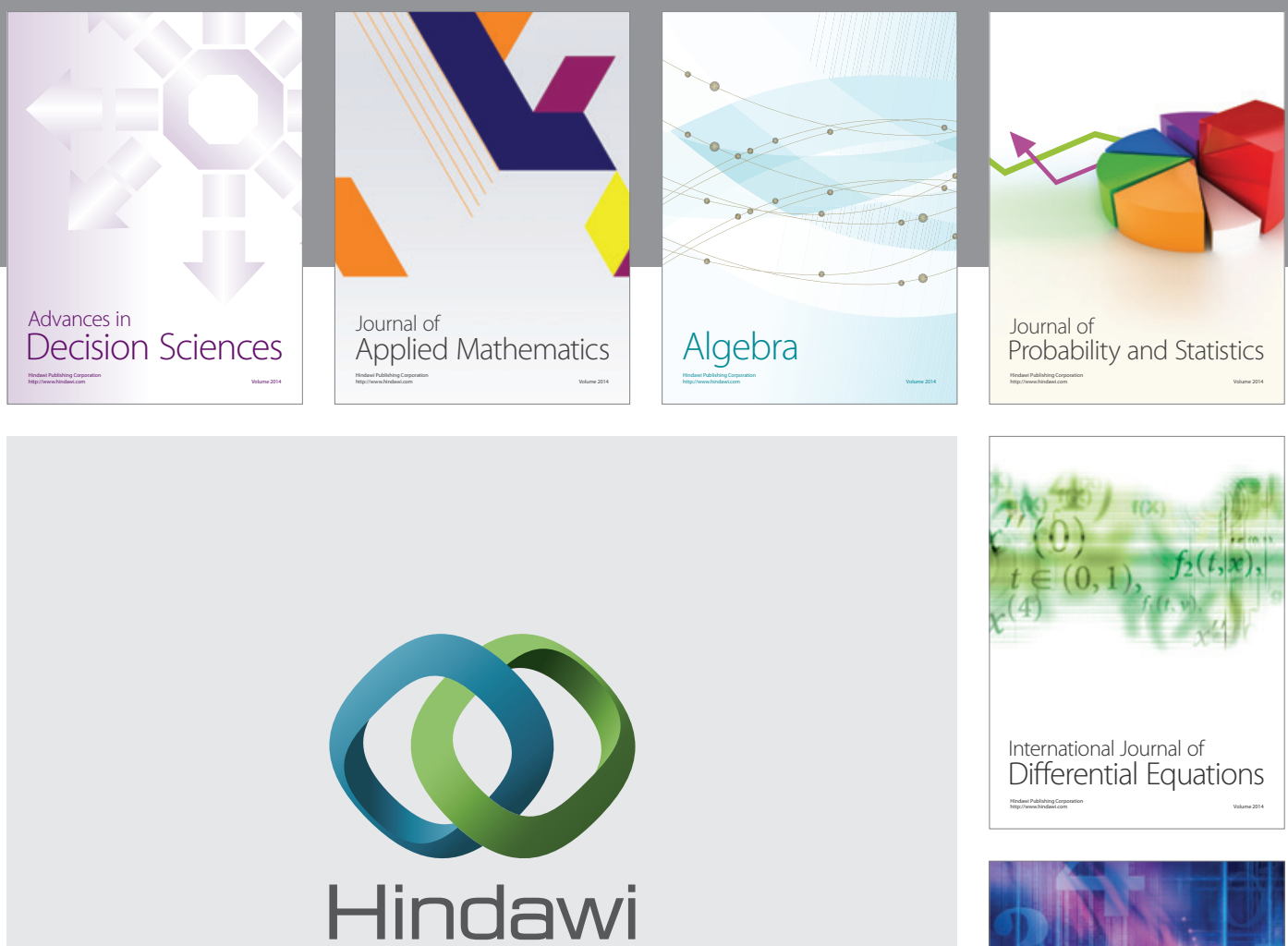

Submit your manuscripts at http://www.hindawi.com
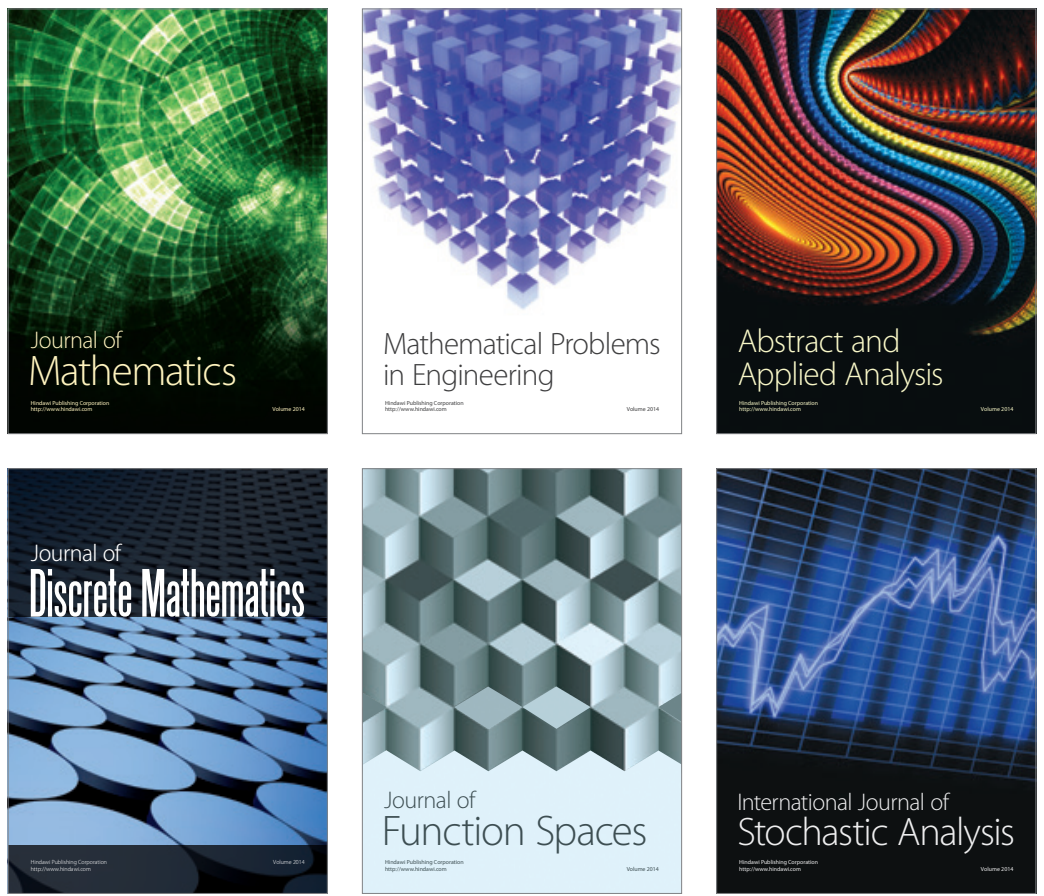

Journal of

Function Spaces

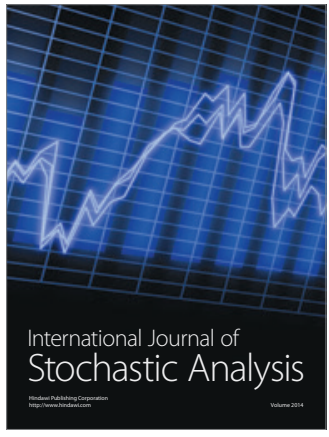

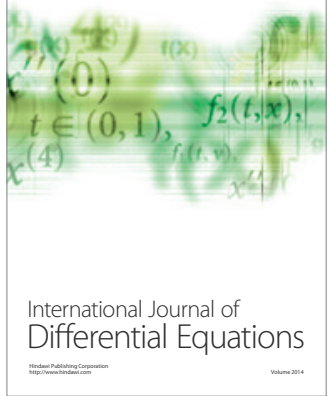
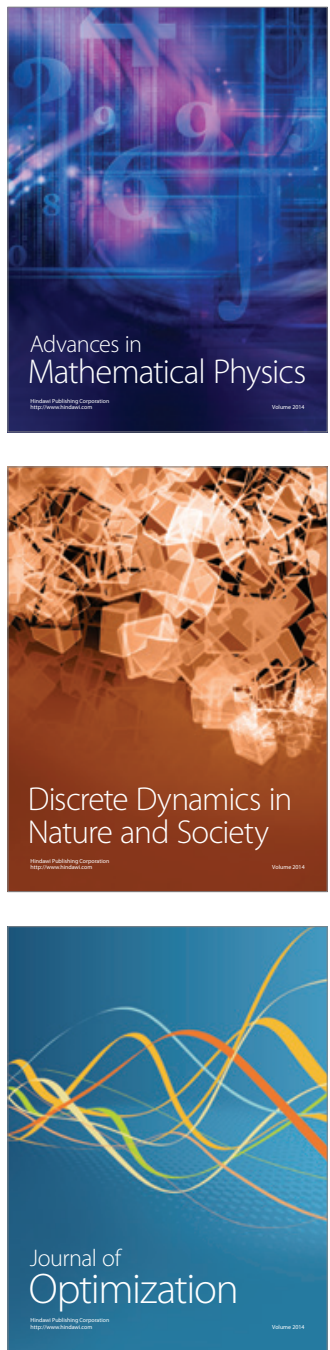